\title{
Anmerkungen zur Grammatik der Wort-Bild-Verbindungen
}

\author{
Andreas Roser, Linz und Passau
}

Funktionalität und Gebrauch sind keine Synonyme. Nicht jeder Sprachgebrauch im Medium der Wortsprache ist funktional. Die Nichtfunktionalität der Wortsprache verweist auf Medienübergänge, Medienverbindungen und Funktionalitätsveränderungen in der Verwendung von Texten. Zweifel am Immanenzcharakter wortsprachlicher Medien sind aus mehreren Gründen angebracht: Wenn in der Wortsprache - wie Wittgenstein es mehrfach formulierte - „alles ausgetragen" ${ }^{1}$ werden könnte, dann wäre beispielsweise ein Bildmedium immer ein Werkzeug eines Textmediums. Dieser Hypothese wird hier widersprochen; unabhängig von der Frage, ob Wittgenstein der Annahme war, alle Medien seien für die Zwecke der Wortsprache instrumentalisierbar und auch unabhängig von der These, es werde „alles“ in der Wortsprache ausgetragen. Dass ,,alles“ in „,der" Sprache ausgetragen wird, wäre freilich wenig zu bezweifeln, sofern wir wüssten, was „alles“ und was „die Sprache“ ist oder auch nur sein könnte. Es gibt etliche Belege für die These, Wittgenstein sei nicht der Auffassung gewesen, Fotos, Zeichnungen, oder musikalische Notationen - um nur Beispiele zu nennen - seien Instrumente der Wortsprache. Es gibt allerdings Belege im Nachlass Wittgensteins, die auf die gegenteilige Annahme hinweisen. Insbesondere die Funktion vieler Piktogramme in Wittgensteins nachgelassenen Schriften lassen sich als Belege für die Instrumentalisierungshypothese visueller Medien anführen; in vielen Fälle allerdings sind es gerade diese Piktogramme, die auch als Belege der gegenteiligen These verwendet werden und eine Autonomie visueller Medienelemente 
anzeigen. Doch das Ziel dieses Vortrages ist nicht eine vorrangige Klärung exegetischer Probleme in den Texten Wittgensteins, sondern eine medienanalytische Klärung der Frage, ob wir nichtverbale Medien als Instrumente der Wortsprache verwenden.

Eines scheint vorab offenkundig zu sein: Verbale Beschreibungen ersetzen keine visuellen Kontexte. Ein Text funktioniert als Text im Medium der Texte. Ein Bild teilt sich im Medium der Bilder mit. Wir stellen mit visuellen Mitteln her, was mit Texten nicht herzustellen ist. Texte sind zwar immer auch visuelle Elemente (darin liegt ihr extraverbaler Gebrauchsaspekt) aber sie funktionieren nicht wie Fotos oder Gemälde. Verhielte es sich anders, könnten wir Gemälde z. B. durch Bildbeschreibungen ersetzen und sie beispielsweise anstelle eines Gemäldes verkaufen. Das wäre zwar möglich, ist aber in der Praxis des Mediengebrauches nicht zu beobachten.

Umgekehrt gilt: Nichtverbale Medien verlieren ihre mediale Funktion in einem verbalen Medium. Mit Worten zu sagen, was nur Bilder unter Bildern auf die Weise eines Bildes mitteilen, wäre der Versuch, Bilder durch Texte zu substituieren. Bilder sind keine Instrumente der Wortsprache; ihre verbale Nichtfunktionalität überträgt sich auf Wort-Bild-Verbindungen. Das ist die These, die zu begründen sein wird. Die These kann darauf verweisen, dass ein Satz auch als visuelles Muster in einer Bildsprache Verwendung finden kann. Als Ornament unter anderen visuellen Mustern verliert ein Satz seine Funktion als Satz unter Sätzen. Der Funktionsverlust eines Satzes ist in diesem Fall aber kein Indikator für einen Gebrauchsfehler der Wortsprache, denn ein Fehler ist nur dort möglich, wo Texte auch Texte verantworten können.

Ein Bild berührt nicht, was in und mit einem Text geschieht, denn wir können aus einem Satz - mit den Mitteln der Wortsprache - keine logischen und grammatischen Regeln für den Umgang mit visuellen Medien ableiten. Ein Bild kann mit einem Text verbunden sein, was immer auch mit einem Satz geschehen mag, der dieses Bild beschreibt. Umgekehrt gilt dies auch von einem Text, der auf unterschiedlichste Weise illustriert werden kann. Weder das Bild noch der Text für sich garantieren eine Medienverbindung. Medienverbindungen sind keine funktionalen Verbindung der beteiligten Medien, sondern ermöglichen den Funktionsübergang von einer Medienverwendung zu einer anderen.

Ein Beispiel: Wir können die Zufallsabfolge der Kugelpositionen in einem Roulettespiel beschreiben und diese Beschreibung könnte mit Fotoprotokollen dokumentiert werden. Die Verbindung des Satzes „Die Kugel liegt auf der 
24" mit einem Foto, das die Kugel auf eben dieser Position zeigt, ist in diesem Falle keine Funktion der verbalen Beschreibung des Bildes. Das Foto ist einer verbale Beschreibung zugeordnet, weil es sich in der Beobachtung eines Ereignisses zufällig so ergeben hat, nicht aber, weil ein Satz der Wortsprache ein Roulettespiel gleichsam nach Regeln des Wortsprachengebrauches steuern könnte. Ein Sprachspiel der Wortsprache regelt nicht die Bedeutung des Satzes „Zufällig liegt die Kugel auf der 24“, denn eine Konvention kann zwar regeln, wie mit Zufallsergebnisse umzugehen ist, aber ein Zufallsergebnis kann sie nicht prognostizieren und auch nicht regelkonform beschreiben, denn es gibt keine Konvention für die Beschreibung eines Zufallsereignisses.

Die mediale Verbindung zwischen dem Satz „Ich sehe die Kugel auf der 24" und einem Foto lässt sich auch nicht aus einem Protokollfoto ableiten. Obwohl wir wissen, was dieser Satz in diesem Kontext bedeutet (Abb.1). Bedeutungsstabilität der Beschreibung einer Zufallsfolge von Ereignissen ist dennoch möglich, weil ein-und-dasselbe Protokoll-Foto für wahre und falsche Aussagen gleichermaßen Verwendung finden kann.

Abb. 1 Beschreibungen und visuelles Protokoll der Position einer Roulettekugel

Beschreibung 1

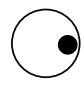

Beschreibung 2

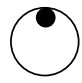

Beschreibung 3

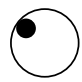

Beschreibung 4

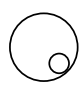

In der Verbindung von Satzzeichen und Fotografien finden wir diese stabilen Bedeutungen, weil Texte und Fotoelemente eine mediale Verbindung eingegangen sind, die nicht in ihren medialen Bestandteilen lag, nicht aus der Herkunft der beteiligten Medienelemente abzuleiten war. Zufallsereignisse wie beispielsweise Roulette-Ergebnisse oder Lottozahlen benötigen für ihre verbale Beschreibung diese Medienschnittstellen. Wir stellen diese Verbindungen zwischen unterschiedlichen Medien her und wir gebrauchen solche Verbindungen, 
aber aus ihrem Gebrauch folgt keine Konvention und keine Konvention folgt diesem Gebrauch; immer vorausgesetzt, wir können einen funktionalen von einem nichtfunktionalen Mediengebrauch unterscheiden. Das entscheidende Argument für die oben angeführte These ist der Nachweis eines nichtfunktionalen Mediengebrauchs, der im angeführten Beispiel an Funktionsverlusten der Wortsprache zu erkennen ist. Solche Funktionsverluste im Gebrauch der Wortsprache sind konstitutiv für Medienübergänge zwischen Texten und Bildern. Ein Satz, der mit einem Foto verbunden ist, funktioniert nicht länger wie ein Satz unter Sätzen. In Verbindung beispielsweise mit einem Foto wird er als etwas verwendet, das sowohl als Bild als auch als Text gelesen werden kann. Ein Satz - als ornamentales Muster betrachtet - verliert seine Funktionalität unter Sätzen; das Foto, als Teil des Satzes verwendet, kann nicht länger wie ein Foto funktionieren, sondern gliedert sich in die Funktionalität des Wortsprachengebrauches ein. Die Verbindung zwischen Texten und Fotos in einem Medienprotokoll ist eine Verbindung unterschiedlicher Gebrauchsformen unterschiedlicher Medien. Sätze, die in eine solche Medienverbindung eintreten, können Übergänge zu beliebigen Bildern herstellen, verändern dadurch aber ihre konventionelle Bedeutung. Dieser Funktionsverlust zeigt eine Grenze der Funktionalität des Medium „Wortsprache“ auf.

\section{Alles wird in Medien ausgetragen}

Otto Neurath beschäftigte sich über viele Jahre hinweg mit dem Projekt, visuelle Botschaften zu entwickeln, die eine bedeutungsstabile und eindeutig verbalisierbare Botschaft enthalten sollten. Neurath ist allerdings nicht der Frage nachgegangen, wie die mediale Verschränkung von visuellen Botschaften und Texten innerhalb eines Textes zu beschreiben wäre. Ludwig Wittgenstein hingegen, in dessen Nachlass sich deutlich mehr als tausend Piktogramme finden, verwendet diese Skizzen und Zeichnungen überwiegend instrumentell, zugleich aber nicht als Instrumente einer Bildsprache. Beiden Philosophen gemeinsam ist die Auffassung, alles werde in der Sprache ausgetragen. Was allerdings unter dieser „Sprache“ zu verstehen ist, wird von ihnen völlig unterschiedlich beantwortet. Für Neurath ist es die Bildsprache, die sich der Wortsprache bedient, und das Leitmedium jeder verbalen Transformation visueller Inhalte ist. Für Wittgenstein hingegen sind Bilder ihrerseits nur Instrumente der Wortsprache: Ein Bild zeigt nur, was mit Sätzen zur 
Darstellung gebracht werden kann. Dennoch lassen beide Konzepte ein gemeinsames Programm erkennen: das Programm der wechselseitigen Übersetzbarkeit von Texten in Bilder und von Bildern in Texte.

Neurath suchte nach einer idealtypischen wortsprachlichen Bedeutung typographischer Bildelemente; der mittlere und späte Wittgenstein hingegen erklärte den Begriff der „Bedeutung“ unter Berufung auf Gebrauchsbedeutungen der Wortsprache, in der Bilder - verbal instrumentalisiert - Werkzeuge der Wortsprache sind.

Beide Ansätze zeigen dennoch ein gemeinsames Thema: Bedeutungsstabilität in Medienübergängen, die weder allein in der Analyse des Wortsprachengebrauches noch in der Analyse des Gebrauchs einer Bildsprache (Fotos unter Fotografien) zu finden ist, sondern ausschließlich in der Verbindung unterschiedlicher Medien und unterschiedlicher Medienelemente. ${ }^{2}$

Dennoch können wir auf geordnete Weise über etwas sprechen, das als visuelles Element dem Gebrauch der Wortsprache nicht zu entnehmen ist. Ein Medium hat die Möglichkeit zu funktionieren oder nicht zu funktionieren nur in Verbindung mit anderen Medien. Es muss also eine Form der Synchronisation geben, um von einem Medium behaupten zu können, es sei mit einem anderen verbunden. Ein Medium ist mit einem anderen synchronisiert, wenn es zu keinen Missverständnissen in der Medienkommunikation kommt. Die Praxis zeigt, dass dies der Regelfall unserer Verständigung ist, wenn wir unterschiedliche Medien zugleich verwenden.

Medienverbindungen, Medienschnittstellen bzw. gemischt-mediale Darstellungen werden von uns im Praxisgebrauch aller Medien auf immer neue Weise hergestellt. Etwas herzustellen setzt aber keine Konvention voraus, sonst könnte nur hergestellt werden, was bereits bekannt ist.

\section{Der nichtfunktionale Wortgebrauch}

Wir finden Wort-Bild-Verbindungen und damit auch mediale Schnittstellen in vielen alltäglichen Situationen, in denn wir mit Worten ein Szenarium beschreiben, das durch visuelle Inhalte zu ergänzen ist. Ein Beispiel für eine solche Medien-Schnittstelle in unserer Alltagskommunikation sind BegrüBungsrituale:

Werden wir einen begrüßen, den wir vielleicht zum ersten Mal an diesem Tag auf der Straße treffen? Werden wir diese Person auch grüßen, 
wenn wir ihr bereits mehrfach an diesem Tag begegnet sind? Wird die geografische Breite, die Wetterlage und die Windrichtung bzw. die Neigungsrichtung des Regenschirms in diesem Begrüßungsritual eine Rolle spielen oder überhaupt die allgemeine körperliche und psychische Verfassung der beteiligten Personen? Welchen Einfluss haben Begleitpersonen des Begrüßten und des Begrüßenden in einer solchen Begrüßungssituation? Wird beispielsweise eine Frau ihren Mann auf der Straße begrüßen, wenn er mit seiner Freundin unterwegs ist - oder umgekehrt?

Die Liste der Situationen des Grüßens und Begrüßtwerdens ist die Liste der in der Wortsprache nicht prognostizierbaren weil kontingenten Einzelfälle. Ist der Zufall des Beschriebenen in der Beschreibung enthalten, dann lässt sich kein allgemeines und auch kein besonderes Merkmal dieses Zufalls benennen oder beschreiben. Dennoch haben wir mit diesen Einzelfällen in der Begrüßungspraxis kein Problem, denn wir visualisieren unsere Worte, inszenieren dieselben verbalen Rituale auf unterschiedliche Weise und verleihen unseren Worten durch diese Visualisierung Gebrauchsstabilität.

Diese Tatsache verlangt eine Erklärung, die sich nicht neuerlich auf kontingente Umstände berufen darf. Welche Grammatik aber verleiht den zahllosen möglichen Begrüßungsritualen die Bedeutungsstabilität? Warum verstehen wir Begrüßungen auch in Situationen, die nicht bekannt sind und nicht antizipiert werden konnten? Eine mögliche Antwort wäre: Die Verbindung eines Satzes mit einem visuellen Inhalt, z. B. auch mit einem Foto, funktioniert weder wie ein Satz noch wie ein Foto, denn eine solche Verbindung kann keine nur verbale und keine ausschließlich visuelle Funktion haben; sie verleiht unseren Worten eine neue Gebrauchsform. Dies mag auch ein Grund dafür sein, warum wir keine Sprachspielverzeichnisse und Regelverzeichnisse des alltäglichen Sprachgebrauchs benötigen, um uns in der Sprache zu orientieren. Wir verwenden ein breites Repertoire von Verhaltensweisen, um starre Medienübergängen herzustellen. Der tatsächliche Sprachgebrauch wird zwischen Medien nicht durch Regelverzeichnisse mit Leben erfüllt, sondern durch Verhaltensweisen, mit deren Hilfe wir Medienschnittstellen herstellen und festlegen. Gebrauchsstabilität liegt in den überall auffindbaren nichtfunktionalen Schnittstellen unterschiedlicher Medien. Wir könnten hier auch von Konventionsverschränkungen sprechen, die selbst die nichtkonventionelle Eigenschaft haben, nämlich neu zu sein und eine Gebrauchsform zu erzeugen, die nicht aus anderen Gebrauchsformen ableitbar ist. 
Die Wortsprache ist eben nicht in allen Fällen einer Werkzeugkiste vergleichbar, in der wir stets und ausnahmslos nach dem passenden Werkzeug für einen bestimmten Zweck suchen. Diese Werkzeugmetapher ist häufig irreführend.

Verliert ein Satz seine Funktionalität in der Wortsprache, bleibt er als visuelles Element (als typografische Zeichenfolge) erhalten. Für Fotografien beispielsweise gilt dies nicht, denn ein Foto kann seine visuelle Mitteilungsfunktion nicht verlieren, es teilt sich selbst mit, auch dann, wenn es seinen Kontext verliert. („Das Bild sagt sich selbst.“3). Ein Bild benötigt keinen visuellen Kontext, um verwendet werden zu können. Von einem Satz hingegen lässt sich nicht behaupten, er teile etwas ,jenseits“ seines sprachlichen Kontextes mit. Bilder funktionieren auch in diesem Falle anders als Texte. Texte, die wir als Bilder (z. B. als typografische Ornamente) betrachten, sind Texte, die ihre ursprüngliche Funktion verloren und die Funktion eines visuellen Mediums übernommen haben. Einen Satz zu verwenden bedeutet deshalb nicht a priori, ihn als Werkzeug der Wortsprache zu verwenden. Wir könnten hier den bekannten naturalistischen Fehlschluss durch den Typus des medialen Fehlschlusses erweitern: Es wäre ein medialer und funktionalistischer Fehlschluss, aus Funktionen des Wortsprachengebrauches auf Funktionen des Bildsprachengebrauches schließen zu wollen.

Wenn Ornamente, Fotos oder andere visuelle Elemente nicht wie Sätze funktionieren, Sätze aber ihrerseits als visuelle Muster betrachtet werden können, dann muss es Sätze geben, die nicht wie Sätze, sondern als visuelle Muster funktionieren. Sätze, die nur so aussehen, als ob sie wie Sätze unter Sätzen funktionieren, verweisen in diesem Fall aber nicht auf sprachlichen oder grammatischen Unsinn, sondern auf Medienverschränkungen, die diesen Sätzen ihre Gebrauchsform entziehen bzw. diese verändern.

Wird ein Satz als visuelles Medium verwendet, verliert er seine Funktion als verbales Medium, muss aber seine Funktion als visuelles Medium in diesem Falle nicht verlieren, denn Sätze sind immer grafische Muster, auch wenn diese Eigenschaft ihre Gebrauchsfunktion innerhalb der Wortsprache nicht maßgeblich bestimmt. Es muss also Sätze und visuelle Elemente in einem Sprachspiel geben, die in der Wortsprache nichtfunktional sind, d.h. in einem Sprachspiel nicht verwendet werden können, damit es Sätze und visuelle Elemente geben kann, die funktional sind bzw. verwendet werden können. Funktionalität und Nichtfunktionalität bedingen sich in der Wortsprache wechselseitig. 
Diese Abgrenzung der Beschreibung der Gebrauchsarten eines Satzes beantwortet aber noch nicht die Frage, woran wir nichtfunktionale Medienelemente erkennen können.

\section{„Sehen als ...“, „,Lesen als ...“}

Gäbe es eine Theorie der unhintergehbaren Funktionalität der Wortsprache, dann hätte man sich mit ihr jeder Möglichkeit beraubt, den Gebrauch eines Satzes im Unterschied zu seinem Nichtgebrauch beschreiben zu können. Eine transzendentalphilosophische Interpretation Wittgensteinscher Sprachspiele wäre nicht in der Lage, Gebrauchsübergänge zwischen Medien zu beschreiben, weil sich ein Medium nicht in ein anderes Medium verwandeln könnte. Die Grenze eines Mediums - in diesem Falle der verbalen Sprache - wäre die Grenze der Sprache überhaupt. Eine transzendentalphilosophische Interpretation der Sprachimmanenz könnte also nicht erklären, warum ein Medium anders funktioniert als ein anderes und warum das, was in einem Medium geschieht, ein anderes Medium nichts angeht.

Innerhalb der Wortsprache scheint die funktionale Differenz zwischen der Sprache und ihrem Beschreibungsgegenstand zwar zu entfallen, denn beschrieben ist, was der Wortgebrauch zu einem Beschriebenen macht. Ein Irrtum aber liegt in der Auffassung, ein Satz verliere jede Funktion, wenn er nicht mehr als Satz unter Sätzen funktioniere. Hier wird die gegenteilige These vertreten: Ein Satz der Wortsprache kann unterschiedliche Funktionen in unterschiedlichen Medien annehmen und andere Funktionen in anderen Medien verlieren. Der wechselnden Medialität, der Medialitätsdynamik der Zeichen, wurde bisher vermutlich zu wenig Aufmerksamkeit geschenkt. Betrachten wir die folgende hybride Medienkonstruktion:

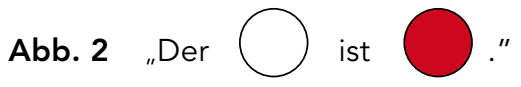

Wir könnten hier von einer gemischt-medialen Mitteilung sprechen, in der wir lesen und sehen können, wovon die Rede ist. Die Gebrauchsnähe unterschiedlicher Medienelemente stellt auch in diesem Fall eine Verbindung zwischen Medienelementen hier, wenn auch zwischen Elementen unterschied- 
licher Medien, zwischen Texten und geometrischen Mustern. Die Frage ist, ob sich diese gemischt-mediale Konstruktion wie ein Satz der Normalsprache analysieren lässt.

„Der ... ist ... . "steht offenkundig nicht für einen analysierbaren Satz der deutschen Sprache.

Auch $\bigcirc$ neben $\bigcirc$ enthält keine Mitteilung, die allein durch diese beiden visuellen Elemente verständlich wäre und dennoch beobachten wir hier eine neue Verbindung von Worten und geometrischen Formen bzw. farbigen Bildelementen. Wir erkennen das Neue dieser Verbindung an der Nichtableitbarkeit dieser Wort-Bild-Verbindung aus dem Gebrauch der Wortsprache. Es gibt keine konventionelle Regel in der Wortsprache, die uns nahelegen würde, diese Konstruktion wie einen Satz der Wortsprache zu behandeln. Es bedarf aber keiner der uns bekannten Grammatiken, um Medienverbindungen dieser und ähnlicher Form in Gebrauch zu bringen bzw. zu verstehen. Wir bringen etwas in Gebrauch, setzen es medial in Verkehr, wenn wir es für den weiteren Gebrauch anbieten, ohne es einem anderen Gebrauch zu entnehmen.

Eine gemischt mediale Grammatik hilft uns zu verstehen, wie grammatische Innovationen entstehen. Auch Patentschriften, die als gemischt-mediale Beschreibungen von Worten und Grafiken weltweit als verbindliche Dokumente gelten, beschreiben Innovationen, ohne sich eines bereits bekannten Gebrauchskontextes bedienen zu können. Die sogenannte „absolute Neuheitserfordernis" schließt von vornherein aus, etwas als Erfindung anzuerkennen, das aus Konventionellem ableitbar wäre oder nur eine Modifikation bekannter technischer Gebrauchsformen enthielte.

Die Nichtfunktionalität eines Textes in technischen Patentschriften finden wir in den neuen Kombinationen der mit dem Text verbundenen Zeichnungen und visuellen Erläuterungen. Diese technischen Wort-Bild-Verbindungen bieten ein hohes Maß an Gebrauchs- und Bedeutungsstabilität, denn Patentschriften müssen international verständlich sein, jenseits aller kulturellen oder regionalen Lebens- und Sprachformen.

Eine Beschreibung technischer Innovationen wird vernünftigerweise nicht nach Konventionen suchen, ein Nichtkonventionelles, weil erfinderisch Neues, konventionell aus bereits Bekannten ableiten zu wollen. Die Nichtfunktionalität eines Mediums in einer neuen Medienverbindung ist eine Voraussetzungsbedingung dafür, die Wortsprache durch andere Medien medial erweitern zu können, ohne „Meta-Medien“ vorauszusetzen. Dass die Wortsprache kein Meta-Medium aller medialen Gebrauchsformen ist, zeigt sich in der 
Abgrenzbarkeit ihrer Gebrauchsformen gegenüber anderen Gebrauchsformen anderer Medien, die ihr, der Wortsprache, keine Rechenschaft schuldig sind. Der Versuch, Medienverbindungen zu beschreiben, führt uns zu Beispielen ihrer Gebrauchsnähe, die verbunden sind mit Funktionsübergängen zwischen den miteinander verbundenen Medien. Vermeintliche Fehler in einem Sprachspiel könnten deshalb in vielen Fällen auch Hinweise auf Medienübergänge und Medienverschränkungen sein. Ob es sich so verhält, kann aber nicht allein durch die Analyse verbaler Sprache gezeigt werden, sondern setzt eine Darstellung des Gebrauches auch anderer mit dem Wortsprachengebrauch synchronisierter Medien voraus. Medien werden synchronisiert verwendet, wenn wir die Medienübergänge herstellen können, ohne uns auf Konventionen berufen zu müssen. Künstlerische und technische Innovationen bedürfen keiner Berufung auf konventionelle Gebrauchsformen eines Mediums. Eine Theorie des innovativen Mediengebrauchs müsste diese Überlegungen an ihren Anfang stellen.

Patentschriften bieten uns klassische Muster der Nichtkonventionalität einer Grammatik bzw. der Gebrauchsform unterschiedlicher Medien, bei gleichzeitig maximaler Klarheit und Deutlichkeit der Beschreibung. Leider fehlt eine Theorie, die uns begreiflich macht, wie das - bei maximaler Nichtkonventionalität - möglich ist bzw. sein kann. Es fehlt uns eine Theorie, die verständlich macht, wie Patentschriften zugleich kommunizierbar und neu sein können.

\section{Anmerkungen}

1 Vgl. MS 156b, p. 6r „Für uns wird alles in der Sprache ausgetragen“; MS 210, p. 36; MS 213, p. 379, p. 388r: „In der Sprache wird alles ausgetragen“. In: Wittgenstein's Nachlass: The Bergen Electronic Edition CD-Rom: Text and Facsimile, Oxford University Press, 2001.

2 Im Sinne einer Arbeitsdefinition können wir ein Medium jede gebrauchsspezifische Mitteilungsform nennen, deren Verwendungsform nur mit den Mitteln dieses Mediums und innerhalb dieses Mediums beschrieben werden kann.

3 Vgl. Wittgenstein „Das Bild sagt mir also sich selbst.“, MS 115, p. 1, 10; MS 146, p. 5, 28, MS 146, p. 28, 63; MS 227a, p. 267; MS 228, p. 113; MS 230, p. 16. In: Wittgenstein's Nachlass: The Bergen Electronic Edition CD-Rom: Text and Facsimile, Oxford University Press, 2001. 